Areej M. Jaber, Jalal A. Zahra*, Mustafa M. El-Abadelah, Salim S. Sabri, Monther A. Khanfar and Wolfgang Voelter*

\title{
Utilization of 1-phenylimidazo[1,5-a]quinoline as partner in 1,4-dipolar cycloaddition reactions
}

https://doi.org/10.1515/znb-2019-0150

Received September 14, 2019; accepted December 8, 2019

\begin{abstract}
A Selected set of 2-(quinolin-2-yl)1,3-oxazepino[7,6- $b]$ indoles $\mathbf{4 a - d}$ and dihydroacenaphtho[1,2-f][1,3] oxazepine $\mathbf{5}$ have been prepared via 1,4-dipolar cycloaddition reaction involving 1-phenylimidazo[1,5-a]quinoline, dimethyl acetylenedicarboxylate, and $N$-(substituted)isatins or acenaphthoquinone. Structures of the new heterocycles $\mathbf{4 a - d}$ and 5 are supported by NMR and HRMS spectral data, and confirmed by single-crystal X-ray crystallography for $\mathbf{4 c}$ and 5.
\end{abstract}

Keywords: 1-phenylimidazo[1,5-a]quinoline; 1,4-dipolar cycloaddition; acenaphthoquinone; dimethyl acetylenedicarboxylate; $N$-methylisatin; $N$-phenylisatin.

\section{Introduction}

Multicomponent reactions (MCRs), in which three or more reactants are connected sequentially in a one-pot reaction, have attracted considerable attention owing to high synthetic efficiency and the facile construction of complex organic molecules [1-13]. 1,4-Dipolar cycloaddition, a subclass of MCRs, emerged as a convenient route towards the synthesis of a variety of six-membered $N$-heterocycles, exemplified by I (Fig. 1). The latter spiroderivative is produced utilizing dimethyl acetylenedicarbxylate (DMAD), pyridine and $N$-methylisatin; herein, the 1,4-dipolar zwitter ion II [14, 15] (Fig. 1) was suggested as transient intermediate arising from nucleophilic addition of the pyridine nitrogen onto DMAD. Interaction of various

\footnotetext{
*Corresponding authors: Jalal A. Zahra, Chemistry Department, Faculty of Science, The University of Jordan, Amman 11942, Jordan, e-mail: zahra@ju.edu.jo; and Wolfgang Voelter, Interfakultaeres Institut fuer Biochemie, Universitaet Tuebingen, Hoppe-Seyler Strasse 4, Tuebingen 72076, Germany, e-mail: wolfgang.voelter@uni-tuebingen.de Areej M. Jaber, Mustafa M. El-Abadelah, Salim S. Sabri and Monther A. Khanfar: Chemistry Department, Faculty of Science, The University of Jordan, Amman 11942, Jordan
}

$N$-heteroarenes and dipolarophiles, together with DMAD, were successfully applied for attainment of the respective spiro-1,3-oxazino heterocycles, and the subject was reviewed [15].

Quite recently, we have applied this reaction in the synthesis of 1,3-oxazepino[7,6-b]indoles III (Fig. 1) utilizing imidazo[1,5-a]pyridines, DMAD and isatins [16]. We sought it would be worthwhile to utilize other $N$-bridgehead-imidazo heterocycles (in placement of imidazo[1,5a]pyridines) so as to widen the scope and applicability of this new reaction towards the synthesis of novel tricyclic and tetracyclic products.

Accordingly, the present work aims at the synthesis of a selected set of model 2-(quinolin-2-yl)-1,3-oxazepino[7,6$b]$ indoles 4a-d and dihydroacenaphtho[1,2-f][1,3]oxazepine 5, utilizing 1-phenylimidazo[1,5-a]quinoline, DMAD, and substituted isatins or acenaphthoquinone as illustrated in Schemes 1-3 (vide infra).

\section{Results and discussion}

\subsection{Synthesis}

The formation of compounds 4a-d most logically proceeds via the intermediacy of the spiro cyclo-adduct [B], produced via cycloaddition of the 1,4-dipolar zwitter ion [A] and the dipolarophilic keto group of the respective isatin (Scheme 2). Adducts [B] then suffer intramolecular skeletal rearrangement involving a cascade of bondbreaking and bond-making processes $([\mathbf{B}] \rightarrow[\mathbf{C}] \rightarrow[\mathbf{D}])$, leading to the respective end products as depicted in the postulated mechanism (Scheme 2). As a consequence, the dihdydroquinoline ring in spiro adducts $[\mathbf{B}]$ restores aromaticity whilst the spiro oxindole entity acquires the status of indolic system conjugated with the maleate moiety; such events represent the driving force for the conversion of the spiro adducts [B] into oxazepino[7,6- $b]$ indoles $\mathbf{4 a - d}$. This mechanism is essentially similar to that previously reported by us for related systems [16].

In its reaction with imidazo[1,5-a]quinoline and DMAD, acenaphthoquinone (an arene-vic-dione) 
<smiles>COC(=O)C1=C(C(C)=O)N2C=CC=CC2OC12C(=O)N(C)c1ccccc12</smiles>

I<smiles>CC=C(C)C(=CC(C)=O)[N+]1CCCCC(C)=[N+]1C(=CC(C)=O)C(C)=O</smiles><smiles>[X]c1ccc2c(c1)c1c(n2[R])OC(c2ccccn2)N(C(=O)[Al])C(C(C)=O)=C1C(C)=O</smiles>

III

Fig. 1: Structures I-III.<smiles>COCC#CCOC</smiles>

1<smiles>c1ccc(-c2ncc3ccc4ccccc4n23)cc1</smiles>

2<smiles>[X]c1ccc2c(c1)C(=O)C(=O)N2[R]</smiles>

$$
\begin{gathered}
\text { 3a-c }(\mathrm{R}=\mathrm{Me}) \\
\text { 3d }(\mathrm{R}=\mathrm{Ph})
\end{gathered}
$$

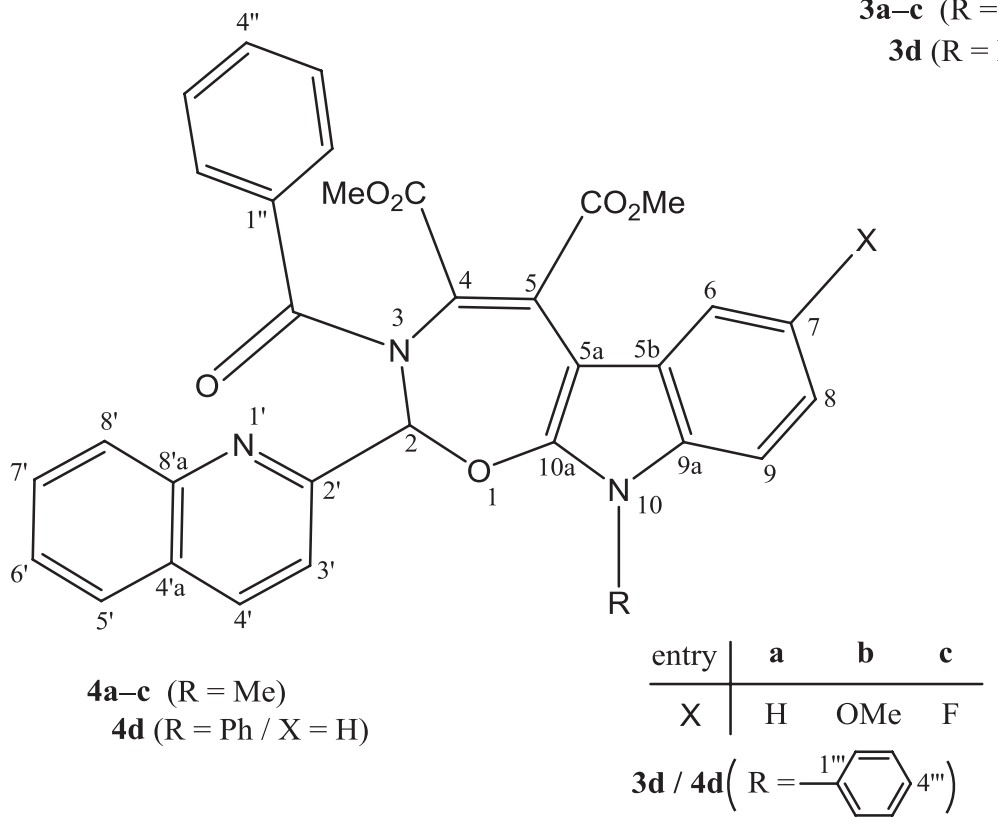

Scheme 1: Formation of 2-(quinolin-2-yl)-1,3-oxazepino[7,6-b]indoles 4a-d.

followed an 1,4-dipolar cycloaddition path similar to that of isatins $\mathbf{3}$ and yielded the respective dihydroacenaphtho[1,2-f][1,3]oxazepine 5 as the end product (Scheme 3).

The new compounds $\mathbf{4 a - d}$ and $\mathbf{5}$ were characterized by MS and NMR spectral data. These data, detailed in the experimental section, are consistent with the suggested structures. Thus, the mass spectra display the correct molecular ion peaks for which the measured high resolution (HRMS) data are in good agreement with the calculated values. DEPT and 2D (COSY, HMQC, HMBC) experiments showed correlations that helped in the ${ }^{1} \mathrm{H}$ and ${ }^{13} \mathrm{C}$ signal assignments to the different carbons and their attached and/or neighboring hydrogens. For compound $\mathbf{4 c}$, the carbons of the benzo-fused ring are readily identified by their doublet signals (with varying $J$ values) 
<smiles>CC(F)=C(F)[n+]1cc2ccc3ccccc3n2c1-c1ccccc1</smiles>

in situ-generated 1,4-dipole

[A]<smiles>[X]c1ccc2c(c1)C(=O)C(=O)N2[R]</smiles><smiles>CC(C)C</smiles>

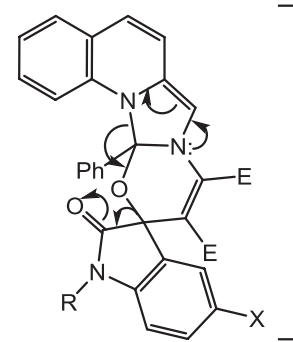

[B]

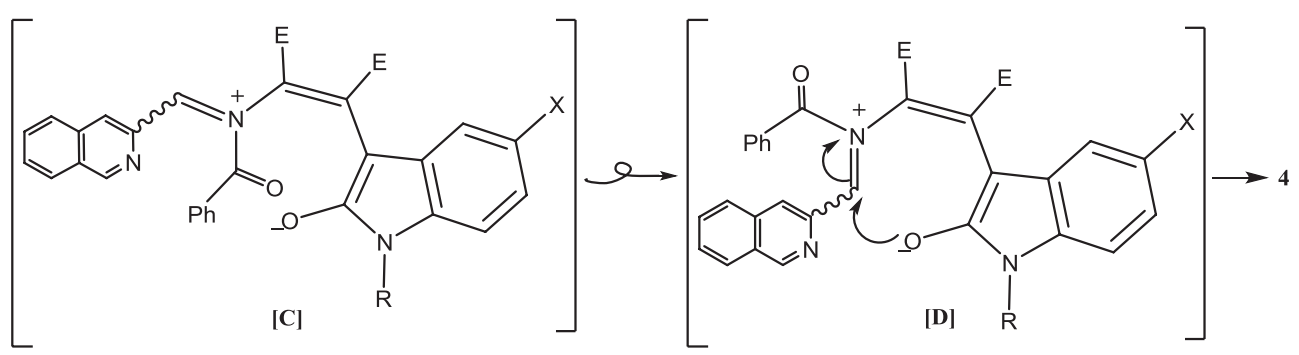

Scheme 2: Postulated mechanism for the formation of oxazepino[7,6-b]indoles $4 a-d$.

$$
\overbrace{\mathrm{CO}_{2} \mathrm{Me}}^{\mathrm{C}}+
$$

1<smiles>Fc1ccccc1-c1ncc2ccc3ccccc3n12</smiles>

2<smiles>O=C1C(=O)c2cccc3cccc1c23</smiles>

acenaphthoquinone

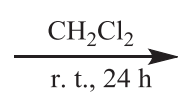<smiles>CC=C(C)C1OC2=C(C(C(C)=O)=C(C(C)=O)N1C(=O)c1ccccc1)c1cccc3cccc2c13</smiles>

5

Scheme 3: Formation of dihydroacenaphtho[1,2-f][1, 3]oxazepine 5.

originating from coupling with the nearby fluorine atom at C-5. Eventually, the structures of 4c (a representative of the present set) and $\mathbf{5}$ are confirmed by single-crystal X-ray crystallography (Figs. 2 and 3; vide infra).

The quinoline ring at $\mathrm{C}-2$ might in part have interesting biological properties to the oxazepino[7,6- $b]$ indoles 4a-d and dihydroacenaphtho[1,2-f][1, 3] oxazepine 5 . This follows from the fact that the quinoline nucleus occurs naturally in quinoline alkaloids with diverse biological activities [17].

\subsection{Molecular structures of $4 \mathrm{C}$ and 5}

Crystal structure determinations were performed to confirm the structures of $\mathbf{4 c}$ and $\mathbf{5}$. Crystal data and a 


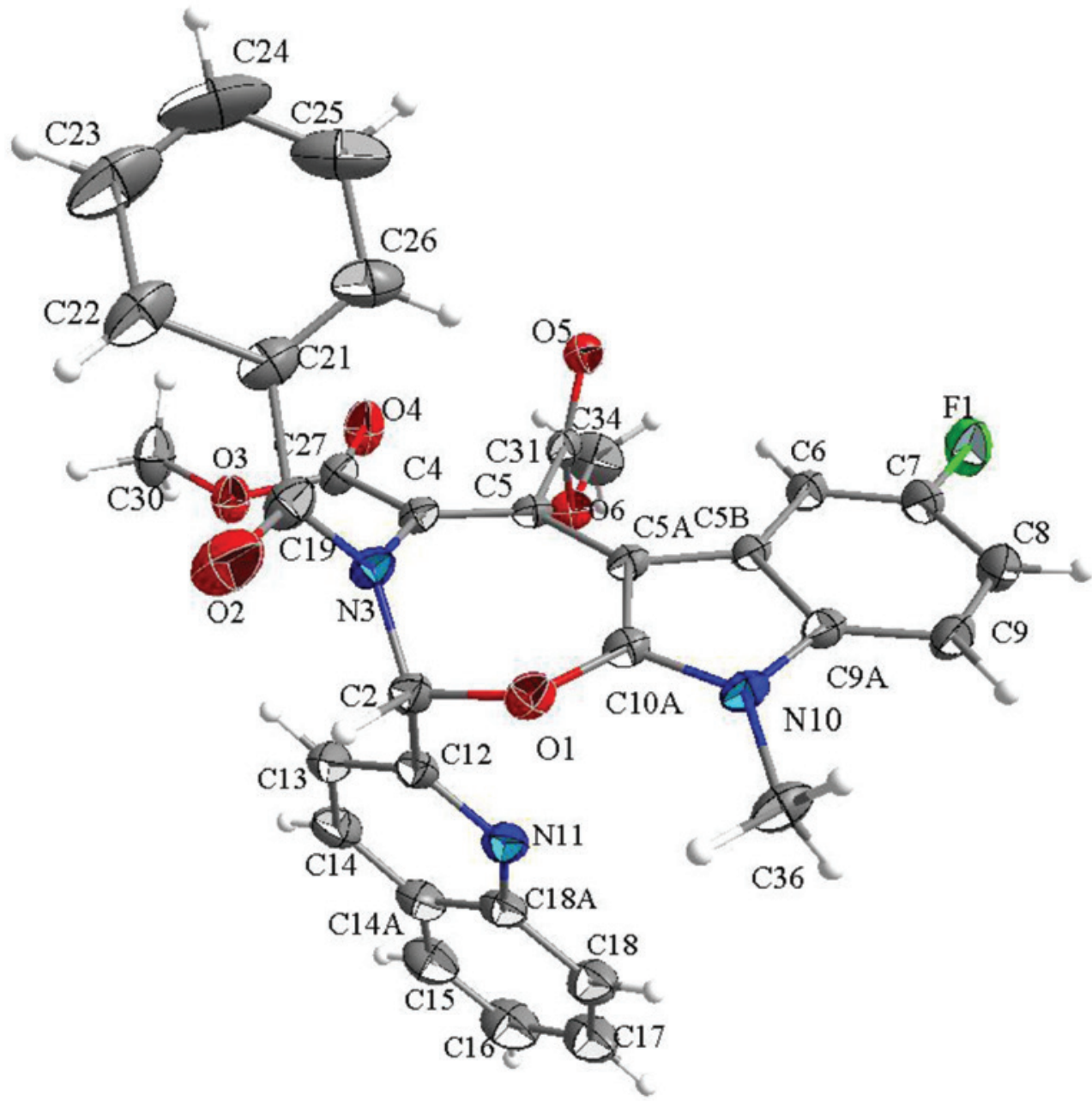

Fig. 2: Molecular structure of $4 \mathrm{c}$ in the crystal (displacement ellipsoids at the $30 \%$ probability level, $\mathrm{H}$ atoms as spheres with arbitrary radii).

summary of data collection and refinement parameters for $\mathbf{4 c}$ and $\mathbf{5}$ are given in Table 1, while selected sets of bond lengths and bond angles for $\mathbf{4 c}$ and $\mathbf{5}$ are provided in Tables 2 and 3, respectively. The molecular structures of $\mathbf{4 c}$ and $\mathbf{5}$ in the crystal are shown in Figs. 2 and 3.

The crystal structure data of compounds $\mathbf{4 c}$ and 5 reveal slight deviations from mean planarity of the sevenmembered rings. The most deviating atoms from planarity are the $s p^{3}$-hydridized $\mathrm{N} 3$ and $\mathrm{C} 2$ atoms with values of -0.539 and $0.267 \AA$ for compound 4c; a comparable deviation is also observed in compound 5 for the $s p^{3}$-hydridized atoms N9 and C8 with values of -0.351 and $0.600 \AA$. A remarkable mutual interaction is observed in compound $\mathbf{4 c}$ between 04 (of the carbonyl group at C4) and C31 of the adjacent carbonyl ester as is evident from the close proximity of the two atoms, with a separation of only 2.58 $\AA$, a value which is $0.640 \AA$ less than the sum of their van der Waals radii. A comparable trend is also observed in compound $\mathbf{5}$ for the atoms $\mathrm{O} 29$ (of carbonyl group at C11) and C32 of the adjacent carbonyl ester, with a separation of only $2.597 \AA$, a value which is $0.623 \AA$ less than the sum of their van der Waals radii. This interaction seems to force both carbonyl esters to acquire almost perpendicular arrangement to one another with a dihedral angle of $87.54^{\circ}$ between $\mathrm{C} 27-04-03-\mathrm{C} 30$ and the $\mathrm{C} 31-05-06-\mathrm{C} 34$ planes for compound $\mathbf{4 c}$, while for compound $\mathbf{5}$ the dihedral angle of $73.45^{\circ}$ is observed between the planes C28O29-O30-C31 and C32-O33-O34-C35.

\section{Experimental section}

Dimethyl acetylenedicarboxcylate (DMAD), quinoline2-carbaldehyde, 5-methoxyisatin, 5-fluoroisatin, $\mathrm{N}$-phenylisatin, acenaphthoquinone and dry dichloromethane were purchased from Acros. (Geel, Belgium). Melting points (uncorrected) were determined on a Gallenkamp electrothermal melting temperature apparatus (London, 


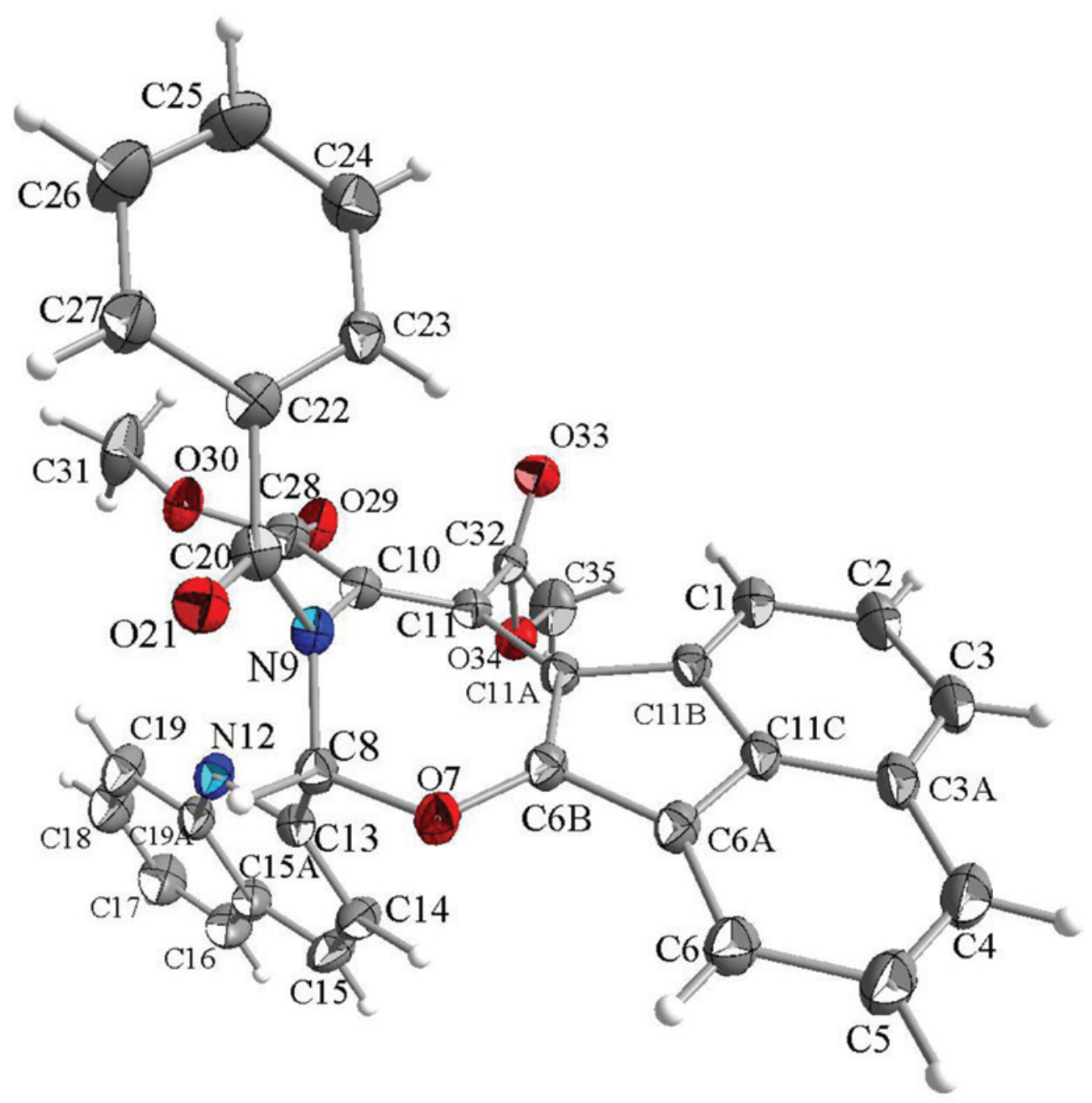

Fig. 3: Molecular structure of $\mathbf{5}$ in the crystal (displacement ellipsoids at the $30 \%$ probability level, $\mathrm{H}$ atoms as spheres with arbitrary radii, co-crystallized molecules EtOAc not drawn).

UK) in open capillary tubes. ${ }^{1} \mathrm{H},{ }^{13} \mathrm{C}$ NMR spectra, and $2 \mathrm{D}$ (COSY, HMQC, HMBC) experiments were recorded on a $500 \mathrm{MHz}$ Bruker Avance-III spectrometer (Bruker, Daltonics, Bremen, Germany) with TMS as internal standard. Chemical shifts are expressed in $\delta$ units; $J$ values for ${ }^{1} \mathrm{H}-{ }^{1} \mathrm{H},{ }^{1} \mathrm{H}-{ }^{19} \mathrm{~F}$, and ${ }^{13} \mathrm{C}-{ }^{19} \mathrm{~F}$ coupling constants are given in Hertz. High-resolution mass spectra (HRMS) were measured using the electrospray ion trap (ESI) technique by collision-induced dissociation on a Bruker APEX-IV (7 Tesla) instrument (Karlsruhe, Germany).

\section{1 $\mathrm{N}$-methylisatin (3a) and 5-fluoro- $\mathrm{N}$ - methylisatin (3c)}

These compounds were prepared by $N$-methylation of the appropriate 5-(substituted) isatin with iodomethane according to a reported procedure [18].

\subsection{1-Phenylimidazo[1,5-a]quinoline (2)}

This compound was prepared by the reaction of quinoline-2-carbaldehyde (in the presence of iodine, potassium bicarbonate and powdered molecular sieves 4 Å) according to a recently reported procedure [19].

\subsection{General procedure for the preparation of 1,3-oxazepino[7,6-b]indoles $4 a-d$ and dihydroacenaphtho[1,2-f][1,3]oxazepine 5}

To a stirred solution of DMAD $(0.71 \mathrm{~g}, 5.0 \mathrm{mmol})$ and substituted isatin or acenaphthoquinone $(5.0 \mathrm{mmol})$ in anhydrous dichloromethane $(15 \mathrm{~mL})$ was added dropwise 1-phenylimidazo[1,5-a]quinoline $(5.0 \mathrm{mmol})$ in dichloromethane $(5 \mathrm{~mL})$ under argon at room temperature. The reaction mixture was stirred for $24 \mathrm{~h}$, the solvent 
Table 1: Crystal data and structure refinement for compounds $4 \mathrm{c}$ and 5.

\begin{tabular}{|c|c|c|}
\hline & 4c & 5 \\
\hline Empirical formula & $\mathrm{C}_{32} \mathrm{H}_{24} \mathrm{FN}_{3} \mathrm{O}_{6}$ & $\mathrm{C}_{35} \mathrm{H}_{24} \mathrm{~N}_{2} \mathrm{O}_{6} \cdot \mathrm{C}_{4} \mathrm{H}_{8} \mathrm{O}_{2}$ \\
\hline Formula weight, $\mathrm{g} \mathrm{mol}^{-1}$ & 565.14 & 656.66 \\
\hline Temperature, $\mathrm{K}$ & \multicolumn{2}{|c|}{ 293(2) } \\
\hline Radiation/wavelength $\lambda, \AA$ & \multicolumn{2}{|c|}{ MoKa/0.71073 } \\
\hline Crystal system & Monoclinic & Triclinic \\
\hline Space group & $P 2_{1} / n$ & $P \overline{1}$ \\
\hline$a, \AA$ & $16.4069(6)$ & $8.1418(13)$ \\
\hline$b, \AA$ & $10.3131(4)$ & $14.856(3)$ \\
\hline$c, \AA$ & $16.6278(6)$ & $15.6311(18)$ \\
\hline$\alpha,{ }^{\circ}$ & 90 & $115.298(14)$ \\
\hline$\beta, \circ$ & $99.935(3)$ & $100.311(12)$ \\
\hline$\gamma,^{\circ}$ & 90 & $95.300(14)$ \\
\hline Volume, $\AA^{3}$ & 2771.33(18) & $1651.2(5)$ \\
\hline$z$ & 4 & 2 \\
\hline Density (calcd.), $\mathrm{g} \mathrm{cm}^{-3}$ & 1.3554 & 1.321 \\
\hline Absorption coefficient $\mu(\mathrm{Mo} K a), \mathrm{mm}^{-1}$ & 0.196 & 0.093 \\
\hline$F(000), e$ & 1176.7 & 688.0 \\
\hline$\theta$ range data collection, ${ }^{\circ}$ & $3.18-29.36$ & $5.944-58.712$ \\
\hline Index ranges $h k l$ & $\begin{array}{l}-20 \leq h \leq 13-14 \leq k \leq 10 \\
-22 \leq l \leq 20\end{array}$ & $\begin{array}{l}-11 \leq h \leq 7 \\
-17 \leq k \leq 18 \\
-21 \leq l \leq 21\end{array}$ \\
\hline Reflections collected & 14810 & 12419 \\
\hline Independent reflections $/ R_{\mathrm{int}}(F)^{2}$ & $6484 / 0.0217$ & $7589 / 0.0445$ \\
\hline Refinement method & \multicolumn{2}{|c|}{ Full-matrix least-squares on $F^{2}$} \\
\hline Data/restraints/parameters & $6484 / 0 / 382$ & $7589 / 0 / 451$ \\
\hline Final $R$ indices $R 1^{\mathrm{a}} / w R 2^{\mathrm{b}}[I>2 \sigma(I)]$ & $0.0488 / 0.1044$ & $0.0915 / 0.2421$ \\
\hline Final $R$ indices $R 1^{\mathrm{a}} / w R 2^{\mathrm{b}}$ (all data) & $0.0846 / 0.1233$ & $0.1493 / 0.3209$ \\
\hline Goodness-of-Fit ${ }^{c}$ on $\left(F^{2}\right)$ & 1.009 & 0.991 \\
\hline Largest diff. peak/hole, $e \AA^{-3}$ & $0.19 /-0.21$ & $0.49 /-0.45$ \\
\hline
\end{tabular}

${ }^{\mathrm{a}} R 1=\Sigma|| F_{0}|-| F \mathrm{c}|| / \Sigma\left|F_{0}\right| ;{ }^{\mathrm{b}} w R 2=\left[\Sigma w\left(F_{0}^{2}-F_{\mathrm{c}}{ }^{2}\right)^{2} / \Sigma w\left(F_{0}^{2}\right)^{2}\right]^{1 / 2}, w=\left[\sigma^{2}\left(F_{0}^{2}\right)+(\mathrm{A} P)^{2}+\mathrm{BP}\right]^{-1}$, where $P=\left(\operatorname{Max}\left(F_{0}^{2}, 0\right)+2 F_{\mathrm{c}}^{2}\right) / 3 ;{ }^{\mathrm{c}} \mathrm{GoF}=S=\left[\Sigma w\left(F_{0}^{2}-F_{\mathrm{c}}^{2}\right)^{2} /\right.$ $\left.\left(n_{\text {obs }}-n_{\text {param }}\right)\right]^{1 / 2}$.

Table 2: Bond Lengths $(\AA \AA)$ and angles $\left(^{\circ}\right)$ for $4 \mathrm{c}$.

\begin{tabular}{lrll}
\hline Bond lengths & \multicolumn{3}{l}{ Bond angles } \\
\hline O1-C10A & $1.3372(19)$ & C10A-O1-C2 & $116.61(12)$ \\
O1-C2 & $1.4296(19)$ & O1-C2-N3 & $110.58(12)$ \\
N3-C2 & $1.452(2)$ & O1-C2-C12 & $111.78(13)$ \\
N3-C4 & $1.423(2)$ & C19-N3-C2 & $117.08(14)$ \\
C5-C4 & $1.356(2)$ & C4-N3-C2 & $119.86(13)$ \\
C5-C5A & $1.437(2)$ & C5-C4-N3 & $120.51(14)$ \\
C10A-C5A & $1.400(2)$ & N3-C4-C27 & $117.80(14)$ \\
C5B-C5A & $1.454(2)$ & C4-C5-C5A & $126.55(15)$ \\
C5B-C9A & $1.410(2)$ & C5A-C5-C31 & $115.65(14)$ \\
N10-C9A & $1.388(2)$ & C5-C5A-C5B & $127.66(14)$ \\
N10-C10A & $1.342(2)$ & C10A-C5A-C5 & $127.73(15)$ \\
N10-C36 & $1.455(2)$ & C6-C5B-C5A & $135.21(15)$ \\
C2-C12 & $1.506(2)$ & 01-C10A-N10 & $115.51(14)$ \\
N3-C19 & $1.379(2)$ & C9-C9A-N10 & $128.77(16)$ \\
C4-C27 & $1.471(2)$ & C10A-N10-C36 & $125.09(15)$ \\
C7-F35 & $1.359(2)$ & O20-C19-N3 & $120.52(17)$ \\
\hline
\end{tabular}

Table 3: Bond lengths $(\AA)$ and angles $\left(^{\circ}\right)$ for 5.

\begin{tabular}{llll}
\hline Bond lengths & \multicolumn{3}{l}{ Bond angles } \\
\hline O7-C6B & $1.333(4)$ & C6B-O7-C8 & $119.1(2)$ \\
O7-C8 & $1.431(3)$ & O7-C8-N9 & $111.3(3)$ \\
N9-C8 & $1.461(4)$ & 07-C8-C13 & $112.3(2)$ \\
N9-C10 & $1.411(4)$ & C20-N9-C8 & $117.2(3)$ \\
C10-C11 & $1.351(5)$ & C10-N9-C8 & $116.6(2)$ \\
C11A-C11 & $1.452(4)$ & C11-C10-N9 & $118.2(3)$ \\
C11A-C6B & $1.383(5)$ & N9-C10-C28 & $120.9(3)$ \\
C11A-C11B & $1.500(5)$ & C10-C11-C11A & $123.8(3)$ \\
C11C-C11B & $1.423(4)$ & C11A-C11-C32 & $116.0(3)$ \\
C11C-C6A & $1.386(5)$ & C11-C11A-C11B & $124.0(3)$ \\
C6A-C6 & $1.379(5)$ & C6B-C11A-C11 & $129.2(3)$ \\
C13-C8 & $1.510(4)$ & C1-C11B-C11A & $137.3(3)$ \\
C28-C10 & $1.482(4)$ & 07-C6B-C6A & $117.9(3)$ \\
N9-C20 & $1.376(4)$ & C6-C6A-C6B & $133.8(4)$ \\
C6A-C6B & $1.456(4)$ & C1-C11B-C11C & $117.3(3)$ \\
O21-C20 & $1.215(4)$ & C6A-C11C-C11B & $111.2(3)$ \\
\hline
\end{tabular}


then removed in vacuo, the residue soaked in $n$-hexane $(10 \mathrm{~mL})$, whereby the desired compound was produced as solid powder; It was then collected by suction filtration, air dried, and purified by chromatographic separation on silica gel plates, eluting with $n$-hexane-ethyl acetate $(4: 1, v / v)$.

\subsection{1 ( $($ )-Dimetyl 3-benzoyl-3,10-dihydro-10-methyl-2- (quinolin-2-yl)-2H-oxazepino[7,6-b]indolo-4,5- dicarboxylate (4a)}

Orange solid; yield: $40 \%$; m.p. $144-145^{\circ} \mathrm{C} .{ }^{-1} \mathrm{H}$ NMR $\left(500 \mathrm{MHz}, \mathrm{CDCl}_{3}\right): \delta=3.46\left(\mathrm{~s}, 3 \mathrm{H}, 5-\mathrm{CO}_{2} \mathrm{CH}_{3}\right), 3.60(\mathrm{~s}, 3 \mathrm{H}$, 4- $\mathrm{CO}_{2} \mathrm{CH}_{3}$ ), 3.97 (s, 3H, N-CH ), 7.10 (pseudo t, $1 \mathrm{H}, 7-\mathrm{H}$ ), 7.30 (pseudo t, 1H, 8-H), 7.31 (d, J=6.9 Hz, 1H, 9-H), 7.36 (pseudo t, 2H, 3"-H/5"-H), 7.38 (t, J=7.2 Hz, 1H, 4"-H), 7.39 (d, J=8.5 Hz, 1H, 3'-H), 7.44 (d, J=7.2 Hz, 1H, 6-H), 7.52 (pseudo t, 1H, 6'-H), 7.56 (d, J=7.2 Hz, 2H, 2"-H/6"'-H), 7.57 (pseudo t, 1H, $7^{\prime}-\mathrm{H}$ ), 7.70 (d, J=8.4 Hz, 1H, 5'-H), 7.72 (d, $\left.J=8.7 \mathrm{~Hz}, 1 \mathrm{H}, 8^{\prime}-\mathrm{H}\right), 8.02\left(\mathrm{~d}, J=8.5 \mathrm{~Hz}, 1 \mathrm{H}, 4^{\prime}-\mathrm{H}\right), 8.35$ (s, 1H, 2-H) ppm. $-{ }^{13} \mathrm{C}$ NMR (125 MHz, $\left.\mathrm{CDCl}_{3}\right): \delta=28.8$ $\left(\mathrm{CH}_{3}-\mathrm{N}\right), 51.6\left(5-\mathrm{CO}_{2} \mathrm{CH}_{3}\right), 52.4\left(4-\mathrm{CO}_{2} \mathrm{CH}_{3}\right), 82.6$ (C-2), 90.9 (C-5a), 108.9 (C-9), 117.9 (C-6), 118.7 (C-3'), 119.1 (C-5),121.9 (C-7), 122.1 (C-8), 123.8 (C-5b), 127.1 (C-5'), 127.4 (C-6'), 127.5 C-4'a), 128.0 (C-3"/5"), 128.1 (C-2"/6"), 129.6 (C-7'), 129.7 (C-8'), 131.2 (C-4"), 133.4 (C-9a), 135.4 (C-1"), 136.6 (C-4), 137.0 (C-4'), 147.0 (C-8'a), 152.6 (C-2'), 154.1 (C-10a), 163.3 (5-CO $\mathrm{Me}$ ), 166.8 (4-CO $\mathrm{Me}$ ), 171.1 (N-COPh) ppm. - HRMS (ESI): $m / z=548.18348$ (calcd. 548.18161 for $\mathrm{C}_{32} \mathrm{H}_{26} \mathrm{~N}_{3} \mathrm{O}_{6}$, $\left.[\mathrm{M}-\mathrm{H}]^{-}\right)$.

\subsection{2 (士)-Dimetyl 3-benzoyl-3,10-dihydro-7-methoxy- 10-methyl-2-(quinolin-2-yl)-2H-oxazepino[7,6-b] indolo-4,5-dicarboxylate (4b)}

Orange solid; yield: 52\%; m.p. $124-126^{\circ} \mathrm{C} .{ }^{-1} \mathrm{H}$ NMR $\left(500 \mathrm{MHz}, \mathrm{CDCl}_{3}\right): \delta=3.41\left(\mathrm{~s}, 3 \mathrm{H}, 5-\mathrm{CO}_{2} \mathrm{CH}_{3}\right), 3.62(\mathrm{~s}, 3 \mathrm{H}$, 4- $\mathrm{CO}_{2} \mathrm{CH}_{3}$ ), 3.77 (s, 3H, 7- $\left.\mathrm{OCH}_{3}\right), 3.84$ (s, 3H, $\left.N-\mathrm{CH}_{3}\right), 6.84$ (d, $J=8.7 \mathrm{~Hz}, 1 \mathrm{H}, 8-\mathrm{H}), 6.90$ (s, 1H, 6-H), 7.17 (d, J=8.7 Hz, 1H, 9-H), 7.31 (pseudo t, 2H, 3"-H/5"-H), 7.36 (t, J=7.0 Hz, 1H, 4"-H), 7.38 (d, J=8.5 Hz, 1H, 3'-H), 7.45 (pseudo t, 1H, 6'-H), 7.52 (d, J=7.3 Hz, 2H, 2"-H/6"'-H), 7.56 (pseudo t, 1H, 7'-H), 7.70 (d, $\left.J=8.0 \mathrm{~Hz}, 1 \mathrm{H}, 5^{\prime}-\mathrm{H}\right), 7.73$ (d, $\left.J=8.5 \mathrm{~Hz}, 1 \mathrm{H}, 8^{\prime}-\mathrm{H}\right)$, $8.03\left(\mathrm{~d}, J=8.5 \mathrm{~Hz}, 1 \mathrm{H}, 4^{\prime}-\mathrm{H}\right), 8.33$ (s, 1H, 2-H) ppm. ${ }^{13} \mathrm{CNMR}$ $\left(125 \mathrm{MHz}_{2} \mathrm{CDCl}_{3}\right): \delta=28.9\left(\mathrm{CH}_{3}-\mathrm{N}\right), 51.4\left(5-\mathrm{CO}_{2} \mathrm{CH}_{3}\right), 52.4$ (4- $\left.\mathrm{CO}_{2} \mathrm{CH}_{3}\right), 55.8$ (7- $\left.\mathrm{OCH}_{3}\right), 82.5$ (C-2), 91.0 (C-5a), 102.7 (C-6), 109.5 (C-9), 110.5 (C-8), 117.9 (C-3'), 118.7 (C-5), 124.4 (C-5b), 127.1 (C-5'), 127.4 (C-6'), 127.5 (C-9a), 128.0 (C-3"/5"), 128.2 (C-4'a), 128.1 (C-2"/6"), 129.6 (C-8'), 129.7 (C-7'), 131.2 (C-4"),
135.5 (C-1"), 136.5 (C-4), 137.0 (C-4'), 147.0 (C-8'a), 152.6 (C-2'), 154.2 (C-10a), 155.6 (C-7), 163.3 (5-CO $\mathrm{Me}$ ), 166.8 (4-CO $\mathrm{Me}$ ), 171.1 (N-COPh) ppm. -HRMS (ESI): $m / z=548.18348$ (calcd. 548.18161 for $\mathrm{C}_{32} \mathrm{H}_{26} \mathrm{~N}_{3} \mathrm{O}_{6}$, [M-H] $]^{-}$).

\subsection{3 (士)-Dimetyl 3-benzoyl-3,10-dihydro-7-fluoro-10- methyl-2-(quinolin-2-yl)-2H-oxazepino[7,6-b] indolo-4,5-dicarboxylate (4c)}

Orange crystal; yield: $47 \%$; m.p. $196-198^{\circ} \mathrm{C} .{ }^{-1} \mathrm{H}$ NMR $\left(500 \mathrm{MHz}, \mathrm{CDCl}_{3}\right): \delta=3.41\left(\mathrm{~s}, 3 \mathrm{H}, 5-\mathrm{CO}_{2} \mathrm{CH}_{3}\right), 3.62(\mathrm{~s}, 3 \mathrm{H}$, 4- $\mathrm{CO}_{2} \mathrm{CH}_{3}$ ), 3.84 (s, 3H, N- $\mathrm{CH}_{3}$ ), 6.94 (pseudo t, $1 \mathrm{H}, 8-\mathrm{H}$ ), $7.05\left(\mathrm{dd}, J_{\mathrm{H}-\mathrm{H}}=2.0 \mathrm{~Hz}, J_{\mathrm{H}-\mathrm{F}}=10.2 \mathrm{~Hz}, 1 \mathrm{H}, 6-\mathrm{H}\right), 7.18(\mathrm{dd}$, $J_{\mathrm{H}-\mathrm{H}}=8.5 \mathrm{~Hz}, J_{\mathrm{H}-\mathrm{F}}=4.0 \mathrm{~Hz}, 1 \mathrm{H}, 9-\mathrm{H}$ ), 7.31 (pseudo t, 2H, 3"$\left.\mathrm{H} / 5^{\prime \prime}-\mathrm{H}\right), 7.37$ (d, J=8.5 Hz, 1H, 3'-H), 7.39 (t, $J=8.8 \mathrm{~Hz}, 1 \mathrm{H}$, 4"-H), 7.43 (pseudo t, 1H, 6'-H), 7.52 (pseudo t, 1H, $7^{\prime}-\mathrm{H}$ ), 7.53 (d, J=6.5 Hz, 2H, 2"-H/6"-H), 7.69 (d, J=7.9 Hz, 1H, 5' $\mathrm{H}), 7.70\left(\mathrm{~d}, J=8.2 \mathrm{~Hz}, 1 \mathrm{H}, 8^{\prime}-\mathrm{H}\right), 8.03\left(\mathrm{~d}, J=8.5 \mathrm{~Hz}, 1 \mathrm{H}, 4^{\prime}-\mathrm{H}\right)$,

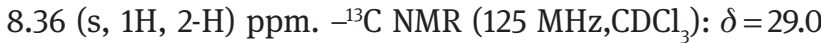
$\left(\mathrm{CH}_{3}-\mathrm{N}\right), 51.6\left(5-\mathrm{CO}_{2} \mathrm{CH}_{3}\right), 52.4\left(4-\mathrm{CO}_{2} \mathrm{CH}_{3}\right), 82.7(\mathrm{C}-2), 91.0$ (C-5a), $105.0 \quad\left({ }^{2} J_{\mathrm{C}-\mathrm{F}}=25.4 \mathrm{~Hz}, \mathrm{C}-6\right), 109.6\left(\mathrm{~d},{ }^{3} \mathrm{~J}_{\mathrm{C}-\mathrm{F}}=9.7 \mathrm{~Hz}\right.$, C-9), $109.7\left({ }^{2} J_{\mathrm{C}-\mathrm{F}}=25.5 \mathrm{~Hz}, \mathrm{C}-8\right), 117.9$ (C-3'), 119.3 (C-5), 124.4 $\left({ }^{3} J_{\mathrm{C}-\mathrm{F}}=10.1 \mathrm{~Hz}, \mathrm{C}-5 \mathrm{~b}\right), 127.2\left(\mathrm{C}-5^{\prime}\right), 127.4$ (C-6'), 127.5 (C-4'a), 128.0 (C-3"/C-5"), 128.1 (C-2"/C-6"), 129.6 (C-7'), 129.8 (C-8'), 131.0 (C-9a), 131.3 (C-4"), 135.5 (C-1"), 136.1 (C-4), 137.1 (C-4'), 146.9 (C-8'a), 152.3 (C-2'), 154.7 (C-10a), $159.1\left({ }^{1} J_{\mathrm{C}-\mathrm{F}}=236.4 \mathrm{~Hz}\right.$, $\mathrm{C}-7), 163.2\left(5-\mathrm{CO}_{2} \mathrm{Me}\right), 166.6$ (4- $\left.\mathrm{CO}_{2} \mathrm{Me}\right), 171.0(\mathrm{~N}-\mathrm{COPh})$ ppm. -HRMS (ESI): $m / z=548.18348$ (calcd. 548.18161 for $\left.\mathrm{C}_{32} \mathrm{H}_{26} \mathrm{~N}_{3} \mathrm{O}_{6},[\mathrm{M}-\mathrm{H}]^{-}\right)$.

Light-orange needle-like crystals suitable for X-ray crystallography were obtained by allowing a warm solution of $\mathbf{4 c}$ in ethyl acetate- $n$-hexane $(1: 1, v / v)$ to stand at r. t. for 2-3 days.

\subsection{4 (士)-Dimethyl 3-benzoyl-3,10-dihydro-10-phenyl- 2-(quinolin-2-yl)-2H-oxazepino[7,6-b]indolo-4,5- dicarboxylate (4d)}

Orange solid; yield: $42 \%$; m.p. ${ }^{130}-132^{\circ} \mathrm{C} .{ }^{-1} \mathrm{H}$ NMR (500 MHz, $\left.\mathrm{CDCl}_{3}\right): \delta=3.49\left(\mathrm{~s}, 3 \mathrm{H}, 5-\mathrm{CO}_{2} \mathrm{CH}_{3}\right), 3.64(\mathrm{~s}, 3 \mathrm{H}$, 4- $\mathrm{CO}_{2} \mathrm{CH}_{3}$ ), 7.10 (pseudo t, 1H, 7-H), 7.18 (pseudo t, $1 \mathrm{H}, 8-\mathrm{H}$ ), 7.24 (d, J=8.4 Hz, 1H, 9-H), 7.35 (pseudo t, 2H, 3"-H/4"-H), 7.41 (t, $\left.J=7.2 \mathrm{~Hz}, 1 \mathrm{H}, 4^{\prime \prime}-\mathrm{H}\right), 7.42$ (d, J=8.2 Hz, 1H, 6-H), 7.44 (d, $J=8.4 \mathrm{~Hz}, 1 \mathrm{H}, 3^{\prime}-\mathrm{H}$ ), 7.49 (pseudo t, 1H, 6'-H), 7.58 (d, J=6.5 Hz, 2H, 2"-H/5"-H), 7.59 (pseudo t, 1H, 7'-H), 7.74 (d, $\left.J=7.4 \mathrm{~Hz}, 1 \mathrm{H}, 5^{\prime}-\mathrm{H}\right), 7.78$ (d, $\left.J=8.3 \mathrm{~Hz}, 1 \mathrm{H}, 8^{\prime}-\mathrm{H}\right), 8.08$ (d, $\left.J=8.4 \mathrm{~Hz}, 1 \mathrm{H}, 4^{\prime}-\mathrm{H}\right), 8.42$ (s, $\left.1 \mathrm{H}, 2-\mathrm{H}\right) \mathrm{ppm} .{ }^{13} \mathrm{C}$ NMR $\left(125 \mathrm{MHz}, \mathrm{CDCl}_{3}\right): \delta=51.7\left(5-\mathrm{CO}_{2} \mathrm{CH}_{3}\right), 52.5\left(4-\mathrm{CO}_{2} \mathrm{CH}_{3}\right), 82.1$ (C-2), 91.5 (C-5a), 110.2 (C-9), 118.0 (C-6), 118.7 (C-3'), 119.8 
(C-5), 122.3 (C-8), 122.4 (C-7), 124.0 (C-5b), 126.9 (C-2"'I/C$\left.6^{\prime \prime \prime}\right), 127.1$ (C-5'), 127.5 (C-6'), 127.6 (C-4'a), 127.6 (C-4'"'), 127.8 (C-3"/5"), $128.1\left(\mathrm{C}-2^{\prime \prime} / 6^{\prime \prime}\right), 128.6\left(\mathrm{C}-3^{\prime \prime \prime} / \mathrm{C}-5^{\prime \prime \prime}\right), 129.6$ (C-7'), 129.7 (C-8'), 131.3 (C-4"), 134.0 (C-9a), 134.9 (C-1'"'), 135.4 (C-1"), 136.3 (C-4), 137.1 (C-4'), 146.9 (C-8'a), 152.3 (C-2'), 153.7 (C-10a), 163.3 (5-CO $\mathrm{Me}), 166.8$ (4- $\left.\mathrm{CO}_{2} \mathrm{Me}\right), 171.0$ (NCOPh) ppm. -HRMS (ESI): m/z=60 818303 (calcd. 60818 271 for $\mathrm{C}_{37} \mathrm{H}_{26} \mathrm{~N}_{3} \mathrm{O}_{6}$, [M-H $]^{-}$).

\subsubsection{Dimethyl 9-benzoyl-8-(quinolin-2-yl)-8,9- dihydroacenaphtho[1,2-f][1,3]oxazepine-10,11- dicarboxylate (5)}

Orange solid; yield: $34 \%$; m.p. $177-179^{\circ} \mathrm{C} . \quad{ }^{1} \mathrm{H}$ NMR $\left(500 \mathrm{MHz}, \mathrm{CDCl}_{3}\right): \delta=3.53\left(\mathrm{~s}, 3 \mathrm{H}, 5-\mathrm{CO}_{2} \mathrm{CH}_{3}\right), 3.62(\mathrm{~s}, 3 \mathrm{H}$, 4- $\mathrm{CO}_{2} \mathrm{CH}_{3}$ ), 7.31 (pseudo t, 2H, 3"- $\mathrm{H} / 5^{\prime \prime}-\mathrm{H}$ ), 7.37 (t, $J=7.2 \mathrm{~Hz}$, 1H, 4"-H), 7.42 (pseudo t, 1H, 2-H), 7.43 (pseudo t, 1H, 6'-H), 7.47 (d, $J=7.2 \mathrm{~Hz}, 1 \mathrm{H}, 1-\mathrm{H}), 7.50$ (d, $\left.J=8.5 \mathrm{~Hz}, 1 \mathrm{H}, 3^{\prime}-\mathrm{H}\right), 7.57$ (d, J=7.5 Hz, 2H, 2"-H/6- $\mathrm{H}^{\prime \prime}$ ), 7.58 (pseudo t, 1H, 7'-H), 7.69 (d, $\left.J=7.9 \mathrm{~Hz}, 1 \mathrm{H}, 5^{\prime}-\mathrm{H}\right), 7.71$ (pseudo t, $\left.1 \mathrm{H}, 5-\mathrm{H}\right), 7.72$ (d, $J=7.8$ $\left.1 \mathrm{H}, 8^{\prime}-\mathrm{H}\right), 7.78(\mathrm{~d}, J=8.4 \mathrm{~Hz}, 1 \mathrm{H}, 3-\mathrm{H}), 7.98$ (d, $J=8.0 \mathrm{~Hz}, 1 \mathrm{H}$, 4-H), 8.00 (d, $\left.J=8.5 \mathrm{~Hz}, 1 \mathrm{H}, 4^{\prime}-\mathrm{H}\right), 8.12$ (d, $\left.J=6.9,1 \mathrm{H}, 6-\mathrm{H}\right)$, 8.44 (s, $1 \mathrm{H}, 8-\mathrm{H})$ ppm. ${ }^{13} \mathrm{C}$ NMR $\left(125 \mathrm{MHz} \mathrm{CDCl}_{3}\right): \delta=51.8$ $\left(10-\mathrm{CO}_{2} \mathrm{CH}_{3}\right), 52.4\left(11-\mathrm{CO}_{2} \mathrm{CH}_{3}\right), 84.6$ (C-8), 109.2 (C-11a), 118.6 (C-3'), 122.5 (C-1), 123.3 (C-6), 125.6 (C-3), 126.3 (C-11c), 126.5 (C-11), 127.1 (C-5'), 127.4 (C-6'), 127.5 (C-4'a), 127.9 (C-3"/C-5"), 128.1 (C-2"'C-6"), 128.1 (C-2), 128.3 (C-3a), 129.4 (C-8'), 129.7 (C-5), 130.7 (C-4), 131.2 (C-4"), 132.6 (C-6a), 135.3 (C-11b), 135.8 (C-1"), 136.6 (C-10), 137.2 (C-4'), 153.8 (C-2'), 162.8 (C-6b), 163.0 (11-CO $\mathrm{Me}), 166.4$ (10-CO $\mathrm{Me}), 171.6$ (N-COPh) ppm. -HRMS (ESI): $m / z=567.15499$ (calcd. 567.15616 for $\left.\mathrm{C}_{35} \mathrm{H}_{23} \mathrm{~N}_{2} \mathrm{O}_{6},[\mathrm{M}-\mathrm{H}]^{-}\right)$.

Red needle-like crystals suitable for X-ray crystallography were obtained by allowing a warm solution of 5 in ethyl acetate- $n$-hexane $(1: 1, v / v)$ to stand at r. t. for 1-2 days.

\section{Collection of X-ray diffraction data and structure analysis of compounds $4 \mathrm{C}$ and 5}

Suitable single crystals of $\mathbf{4 c}$ (with approximate dimensions of $0.08 \times 0.04 \times 0.02 \mathrm{~mm}^{3}$ ) and 5 with approximate dimensions of $0.08 \times 0.06 \times 0.02 \mathrm{~mm}^{3}$ were epoxy-mounted on glass fibers. Data for $\mathbf{4 c}$ and $\mathbf{5}$ were then collected at room temperature $(T=293 \mathrm{~K})$ using an Oxford Calibur Diffractometer. Data were acquired and processed to give SHELX-format $h k l$ files using CRYSALISPRo software
[20]. Cell parameters were determined and refined using CrysAlisPro [20]. A multiscan absorption collection was applied with maximum and minimum transmission factors of 1.000 and 0.929 , and 1.000 and 0.893 for compounds 4c and 5, respectively. The structures were solved by Direct Methods and refined by full-matrix least-squares on $F^{2}$ using all unique data [21]. All non-hydrogen atoms were refined anisotropically with the hydrogen atoms placed in calculated positions and refined using a riding model.

CCDC 1917375 and 1952187 contain the supplementary crystallographic data for compounds $\mathbf{4 c}$ and 5, respectively. These data can be obtained free of charge from The Cambridge Crystallographic Data Centre via www.ccdc. cam.ac.uk/data_request/cif.

\section{Supporting information}

${ }^{1} \mathrm{H}$ and ${ }^{13} \mathrm{C}$ NMR spectra of representative compounds 4a (Figs. S1-S11), 4b (Figs. S12-S23), 4c (Figs. 24-S34), 4d (Figs. S35-S46) and 5 (Figs. S47-S58) are given as supplementary material available online (DOI: 10.1515/ znb-2019-0150).

Acknowledgements: This research work has been supported financially by the Scientific Research Support Fund (SRSF/project number-Bas/2/13/2016) at Amman, Jordan.

\section{References}

[1] I. Ugi, A. Dömling, W. Hörl, Endeavour 1994, 18, 115-122.

[2] H. Bienaymé, C. Hulme, G. Oddon, P. Schmitt, Chem. Eur. J. 2000, 6, 3321-3329.

[3] J. Zhu, H. Bienaymé (Eds.), Multicomponent Reactions, Wiley$\mathrm{VCH}$, Weinheim, 2005.

[4] B. Ganem, Acc. Chem. Res. 2009, 42, 463-472.

[5] E. Ruijter, R. Scheffelaar, R. V. A. Orru, Angew. Chem. Int. Ed. 2011, 50, 6234-6246.

[6] G. M. Ziarani, N. Lashgari, F. Azimian, H. G. Kruger, P. Gholamzadeh, ARKIVOC 2015 (IV), 1-139.

[7] A. Dömling, I. Ugi, Angew. Chem. Int. Ed. 2000, 39, 3169-3210.

[8] A. Dömling, Curr. Opin. Chem. Boil. 2000, 4, 318-323.

[9] M. D. Burke, S. L. A. Schreiber, Angew. Chem. Int. Ed. 2004, 43, 46-58.

[10] D. Ramón, M. Yus, Angew. Chem. Int. Ed. 2005, 44, 1602-1634.

[11] A. Dömling, Chem. Rev. 2006, 106, 17-89.

[12] T. Kaur, P. Wadhwa, S. Bagchi, A. Sharma, Chem. Commun. 2016, 52, 6958-6976.

[13] S. Kundo, B. Basu, RSC. Adv. 2015, 5, 50178-50185. 
[14] R. Huisgen in Topics in Heterocyclic Chemistry (Ed.: R. Castle), John Wiley and Sons, New York, 1969, chapter 8, p. 223.

[15] V. Nair, A. Deepthi, D. Ashok, A. E. Raveendran, R. J. Paul, Tetrahedron 2014, 70, 3085-3105

[16] M. S. Sammor, A. Q. Hussein, F. F. Awwadi, M. M. El-Abadelah, Tetrahedron 2018, 74, 42-48.

[17] P.-Y. Chung, Z.-X. Bian, H.-Y. Pun, D. Chan, A. S.-C. Chan, C.-H. Chui, J. C.-O. Tang, K.-H. Lam, Future Med. Chem. 2015, 7, 947-967.

[18] F. Auria-Luna, E. Marques-Lopez, S. Mohammadi, R. Heiran, R. P. Herrera, Molecules 2015, 20, 15807-15826.
[19] Q. Wang, S. Zhang, F. Guo, B. Zhang, P. Hu, Z. Wang, J. Org. Chem. 2012, 77, 11161-11166.

[20] CRYSAlis Pro Software System (version 1.171.35.11), Intelligent Data Collection and Processing Software for Small Molecule and Protein Crystallography, Rigaku Oxford Diffraction, Yarnton, Oxfordshire (U.K.) 2011.

[21] G. M. Sheldrick, SHelXtL (version 6.10), Bruker AXS Inc., Madison, Wisconsin (USA) 2002.

Supplementary Material: The online version of this article offers supplementary material (https://doi.org/10.1515/znb-2019-0150). 